\title{
Three Common Misconceptions about Visual Impairments
}

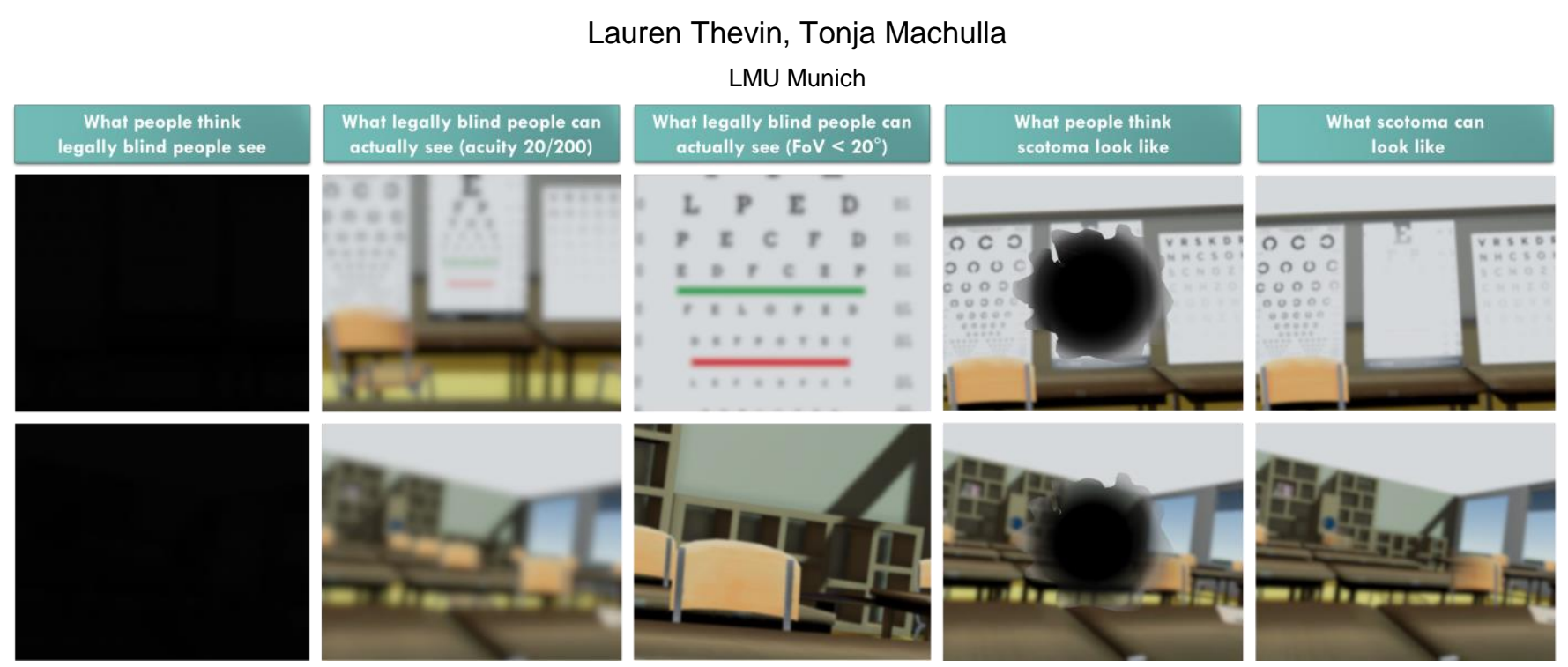

Figure 1. On the top left: a common misunderstanding is that legally blind people do not see anything at all. In the center, a simulation with an acuity considered as legally blind in the USA. On the right, the percept from the same point of view with a reduced field of view (FoV) [5]. On the bottom left, a typical representation of scotoma. On the right, a simulation of auto-completion, where the brain reconstructs the missing part of the image (but fails to reconstruct text and some chairs).

\section{ABstract}

In this work, we address three common misconceptions about visual impairments with an interactive virtual reality simulation. We raise awareness of the phenomenological consequences of central and peripheral field defects and the remaining visual potential of "legally blind" individuals. The goal is to sensitize the public to the effects of visual impairments on daily life activities.

Keywords: Visual impairments, impairment simulation, sensitization.

Index Terms: [Systems, man, and cybernetics]: User interfaces Human computer interaction; [Electronic design automation and methodology]: Design methodology_Graphics_-Virtual reality

\section{INTRODUCTION}

Misconceptions about disabilities can lead to serious issues. In case of visual impairment, poor understanding of the phenomenological consequences of blindness and low vision can lead to verbal and physical aggression towards affected individuals (e.g., [2]). A person who recognizes an acquaintance on the street but fails to shake an extended hand may be perceived as impolite, and a person who uses a white cane for walking but then sits down to read the newspaper may be perceived as a malingerer. In fact, both are symptoms of a severely decreased field of view, or tunnel vision. In even more extreme cases, rich visual hallucinations, which are common in visually deprived individuals and are termed Charles Bonnet Syndrome, may result in mentally healthy individuals being referred to a psychiatrist [1].

Various techniques and simulation tools exist to sensitize family members and medical professionals to difficulties resulting from vision impairments; however, low realism of the sensitization procedure can lead to a lack of understanding of specific behavioral phenomena in everyday life [3]. Here, we simulate visual impairment to counteract three frequent misconceptions that are held by many sighted people and often creep into demonstrations and simulations (Fig. 1):

Misconception 1 (blindness): legally blind people cannot see anything-they live in "darkness",

Misconception 2 (central scotoma): in case of central vision loss, a "black" spot is visible in the center of the vision and affected individuals are always aware that they are missing information,

Misconception 3 (tunnel vision): in case of peripherical vision loss, a "black" surrounding is visible around the center of vision and affected individuals are always aware that they are missing information.

In our proposed simulations the impairments are perceptually not or less obvious than in typical representations, which makes their behavioral consequences more startling.

\section{Phenomenological study of Visual impairments}

We worked in collaboration with a school for students with visual impairments. Four professionals at the school (autonomy and mobility instructors, orthoptists) identified misconceptions regarding visual impairments, typical for relatives and professionals working with people with visual impairments. Here, we focus on three common issues.

The first misconception likely results from the term "legally blind". This can denote a person with a visual acuity of $20 / 200$ or a visual field of less than 20 degrees in USA. Blindness without light perception, i.e. total absence of vision, is rare. There are few official statistics of the prevalence of total blindness, but it is generally estimated that about $90 \%$ of legally blind people have some form of light perception. For instance, in [4], 5.7\% of the blind participants were totally blind (32 out of 561 ), and $6 \%$ were totally blind of one eye (34 out of 561). Including people with low vision, an estimated $99 \%$ of people with visual impairments have residual visual function.

The second and third misconceptions are that partial vision loss (scotoma) necessarily results in perceptually black areas within the visual field and that the affected person is aware of the information 

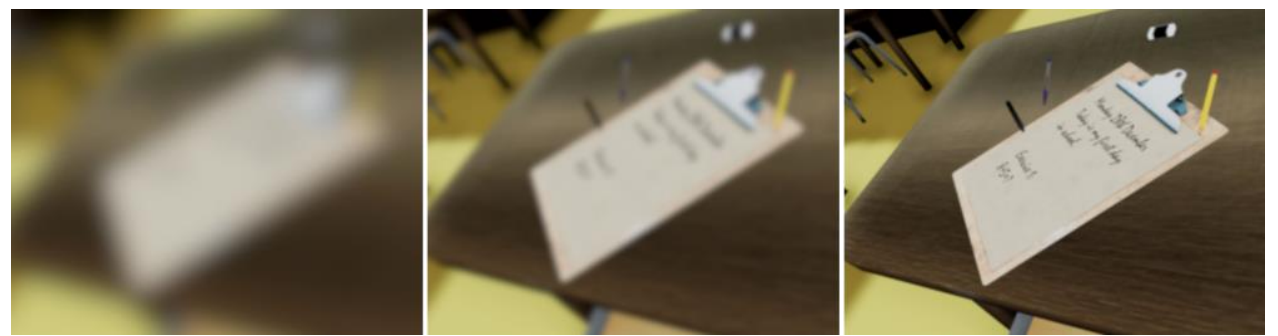

Figure 2. The continuum of visual impairment (acuity). On the left, only a clipboard is identified. The second picture reveals one yellow pencil, a back pencil and the text. The text is readable in the last picture as well as a third blue pen. While the acuity is affected by blurred vision [7], knowledge of the environment helps. Looking back to the left, note that, once you know an object exist, it becomes easier to "see" or "guess" where it is. This demonstrates that perception of the same scene can vary.

loss suffered at these locations. However, there is evidence that individuals with a central scotoma may experience autocompletion, a phenomenon where the cognitive system extrapolates visual information from the surrounding area into a blind spot [6]. Thus, a loss of central vision may not always be obvious.

Similarly, in tunnel vision the vision loss in the periphery may not be experienced as a black area surrounding the functional field of view. The phenomenological experience is rather akin to how fully sighted people visually perceive space outside their field of view (e.g., space behind their heads)-namely, not as a black field but rather there is no visual experience at all.

\subsection{Approach}

Virtual reality and eye-tracking enable features not possible with current sensitization practices such as visual defects that move with the fixation of the eyes, auto-completion mechanisms, or perceptual multiplicity of situations at the same physical location.

We use the HTC Vive Pro with an integrated eye tracker and handheld controllers. We used Unity to develop three simulations regarding acuity, tunnel vision, and central scotoma.

Acuity is modified with a post processing blur effect on the camera. The eye chart is used to set different levels of visual acuity. An exploration of the surrounding environment reveals the associated perceptual experience. It becomes apparent that individuals categorized as "legally blind" can have a comprehensive perception of coarse structures, assisting them in tasks that require spatial orientation and identification of objects and persons.

Tunnel vision is complicated to recreate in virtual reality without creating strong cybersickness. To create tunnel vision without modifying the field of view of the virtual camera nor making the tunnel vignette too obvious, we use two mechanisms. First, we exploited the reduced field of view in VR headset $\left(110^{\circ}\right)$, a fact that is often not apparent to users, and second, we add smooth tunnel vision (blur edge vignette). The consequences of this perceptual modification become evident when walking-the user has a vague sense of postural instability while retaining good central vision. To simulate a central scotoma, we modify the textures of the objects. In VR, due to stereoscopic vision, filters at the camera may appear as floating objects. To avoid this effect, we modify the appearance of the object itself. The objects have different textures: the main texture, and the scotoma texture. When the object is fixated, the scotoma texture replaces the main texture. It provides low detail vision or can even be transparent (objects disappear). The shape and size of the scotoma is defined by another texture: the blind spot texture. This texture is positioned on the screen (or camera) coordinate. The transparency of this texture defines the blending between the scotoma and the main textures. The fully transparent part is in the middle of the image (scotoma texture only) and gradually becomes opaque (main texture only). The scotoma follows the eye tracking by changing the tiling of the texture in the screen coordinates.

Since handicaps result from interaction with the environment, the users perform daily life tasks in a virtual living room, kitchen, street and classroom, interacting with objects through the Vive controllers. Through headset tracking, body positioning and size is maintained consistent with the user body to support embodiment (seven subscales of VEQ [8]). A child's perspective is modelled in the virtual classroom, possibly fostering Proteus effect [9].

\section{LimitATIONS AND FUTURE WORK}

When simulating visual impairments, it is an open question the effect of sense of embodiment is an open question, as the effect of vision is often involved, starting from rubber hand illusion [10]. The current work will be extended to include a broader range of symptoms, such as photophobia or Charles Bonnet syndrome. Moreover, the simulation does not yet include interactions with other people. Finally, many solutions, in particular regarding the environment, exist to improve accessibility. Such approaches are only partially integrated into the demonstration and will be included in later work to ensure awareness regarding disabilities. Acknowledgement: School IRSA-Alfred Peyrelongue (France)

\section{REFERENCES}

[1] Kester, Elizabeth M. (2009). Charles Bonnet syndrome: case presentation and literature review. Optometry-Journal of the American Optometric Association, vol. 80, no 7, p. 360-366.

[2] https://www.bbc.com/news/blogs-trending-47031509

[3] Silverman, A. (2015). The perils of playing blind: problems with blindness simulation and a better way to teach about blindness. Journal of Blindness Innovation and Research, 5(2).

[4] Shah, S. P., Minto, H., Jadoon, M. Z., Bourne, R. R., Dineen, B., Gilbert, C. E., \& Khan, M. D. (2008). Prevalence and causes of functional low vision and implications for services: the Pakistan National Blindness and Visual Impairment Survey. Investigative ophthalmology \& visual science, 49(3), 887-893.

[5] https://www.ncbi.nlm.nih.gov/books/NBK448182/

[6] Bender, Carsten/Schnurnberger, Marion (2017): Zwischen Sehen und Nicht-Sehen. Eine wahrnehmungs- und lebensweltanalytische Ethnographie zur Situation von Menschen mit Sehbeeinträchtigung im Alter. Weinheim/Basel: Beltz Juventa

[7] Krösl, K., Bauer, D., Schwärzler, M., Fuchs, H., Suter, G., \& Wimmer, M. (2018). A VR-based user study on the effects of vision impairments on recognition distances of escape-route signs in buildings. The Visual Computer, 34(6-8), 911-923.

[8] Roth, D., \& Latoschik, M. E. (2019). Construction of a Validated Virtual Embodiment Questionnaire. arXiv preprint arXiv:1911.10176.

[9] Yee, N., \& Bailenson, J. (2007). The Proteus effect: The effect of transformed self-representation on behavior. Human communication research, 33(3), 271-290.

[10] Kilteni, K., Groten, R., \& Slater, M. (2012). The sense of embodiment in virtual reality. Presence: Teleoperators and Virtual Environments, 21(4), 373-387. 